\title{
Implementation Experience of a Patient Monitoring Solution based on End-to-End Standards
}

\author{
I. Martínez, J. Fernández, M. Galarraga, Student Member IEEE, L. Serrano, Senior Member IEEE, \\ P. de Toledo, J. Escayola, S. Jiménez-Fernández, S. Led, M. Martínez-Espronceda and J. García
}

\begin{abstract}
This paper presents a proof-of-concept design of a patient monitoring solution for Intensive Care Unit (ICU). It is end-to-end standards-based, using ISO/IEEE 11073 (X73) in the bedside environment and EN13606 to communicate the information to an Electronic Healthcare Record (EHR) server. At the bedside end a plug-and-play sensor network is implemented, which communicates with a gateway that collects the medical information and sends it to a monitoring server. At this point the server transforms the data frame into an EN13606 extract, to be stored on the EHR server. The presented system has been tested in a laboratory environment to demonstrate the feasibility of this end-to-end standardsbased solution.
\end{abstract}

\section{INTRODUCTION}

$I^{\top}$ TENSIVE Care Units (ICUs) have been in last decades [1]-[3] the main bedside environment of hospitals where advances in technology have implied important changes in the Medical Devices (MDs), computers and sensors at the Point of Care (PoC). These devices acquire huge amounts of very valuable information, without the need for manually writing down each measurement, contributing to solutions based on the Electronic Healthcare Record (EHR) [4]-[6]. The communications within components of a patient monitoring system, and inter-system coordination become now very important in exploiting all the possibilities offered by the information gathered [7]-[9]. However, different manufacturers use their own software and communication protocols; building proprietary solutions that can only work alone or inside single-vendor equipment.

As pointed out at the EMBS06 [10], a standardized communication framework is necessary in order to solve the interoperability problem that now emerges. Two of the standards with research interest nowadays are ISO/IEEE 11073 for PoC-MDs Communications (also known as X73) [11], and EN13606 for EHR communication [12]; a brief overview can be found in [13]. X73 is a family of standards

This research work has been partially supported by projects TSI2005-07068-C02-01 and TSI2004 04940-C02-01 from Ministerio de Educación y Ciencia (Spanish Government), and a personal grant to both M.Galarraga and M. MartinezEspronceda from Navarre Regional Government. I.Martínez, J.Fernández, J.Escayola and J.García are with the Communications Technologies Group (GTC) - Aragon Institute for Engineering Research (I3A) - Univ. Zaragoza (UZ), Spain (e-mail: imr@unizar.es). M.Galarraga, L.Serrano, S.Led and M.Martínez-Espronceda are with the Electrical and Electronics Engineering Dept. - Public Univ. Navarre (UPN), Spain (e-mail: miguel.galarraga @unavarra.es). P. de Toledo and S.Jiménez-Fernández are with the Bioengineering and Telemedicine Research Centre (GBT) - Technical Univ. Madrid, Spain. P. de Toledo is now Visiting Professor at the Informatics Dept. - Carlos III Univ., Spain. (e-mail: paula @gbt.tfo.upm.es). for MDs interoperability. EN13606 is the European standard for EHR aiming to provide health care services in any part of the European Union. As opposed to other standards in the domain (i.e. HL7), the standard defines the architecture of an EHR network instead of a closed hospital environment. To the best of our knowledge there is no experience where the full chain from bedside monitoring devices to EHR has been implemented using standards. Even though there have been some initiatives to combine different standards, the vision of an entire end-to-end standard system is not yet a fact.

The system presented in this paper is a proof-of-concept design to show that it is feasible to create a patient monitoring solution for ICU environments that is end-to-end standards-based. Thus, ISO/IEEE11073(X73) has been used in the bedside environment and EN13606 to communicate the information to an EHR server. The components of this system (sensors, gateway, EHR server, etc.) can be replaced individually by equivalent standards-based devices with no need of system reconfiguration. In the case of bedside monitoring devices, this interoperability is plug-and-play.

This work aims to generate know-how and implementation guidelines to be used in future developments and to detect requirements that have not been addressed up to now. Feed-back will analyze how these implementations must be adapted to follow the future coming standard changes and how the standard can be improved to better fit real-world needs, especially keeping in mind telemedicine and home monitoring scenarios.

The paper is organized as follows. First, an overview of the architecture and design of the proposed system is given in Section II. The implementation experience according to X73 and EN13606 standards is detailed in Section III. The results of the implementation are discussed in Section IV.

\section{II.SYSTEM ARCHITECTURE AND DESIGN}

Figure 1 shows the proposed system architecture, including the fully standards-based prototype, which is made of independent interoperable modules. The generic design consists of several X73 MDs in the PoC related to the ICU. The information collected from these devices is integrated into a gateway. These X73 gateways from each PoC (even from others X73-sensors networks) interconnect with the monitoring server to manage the e-Health service. This monitoring server can also send the acquired patient information to the EHR server according to the EN13606 standard. The specific technical characteristics of each architecture element are following detailed. 


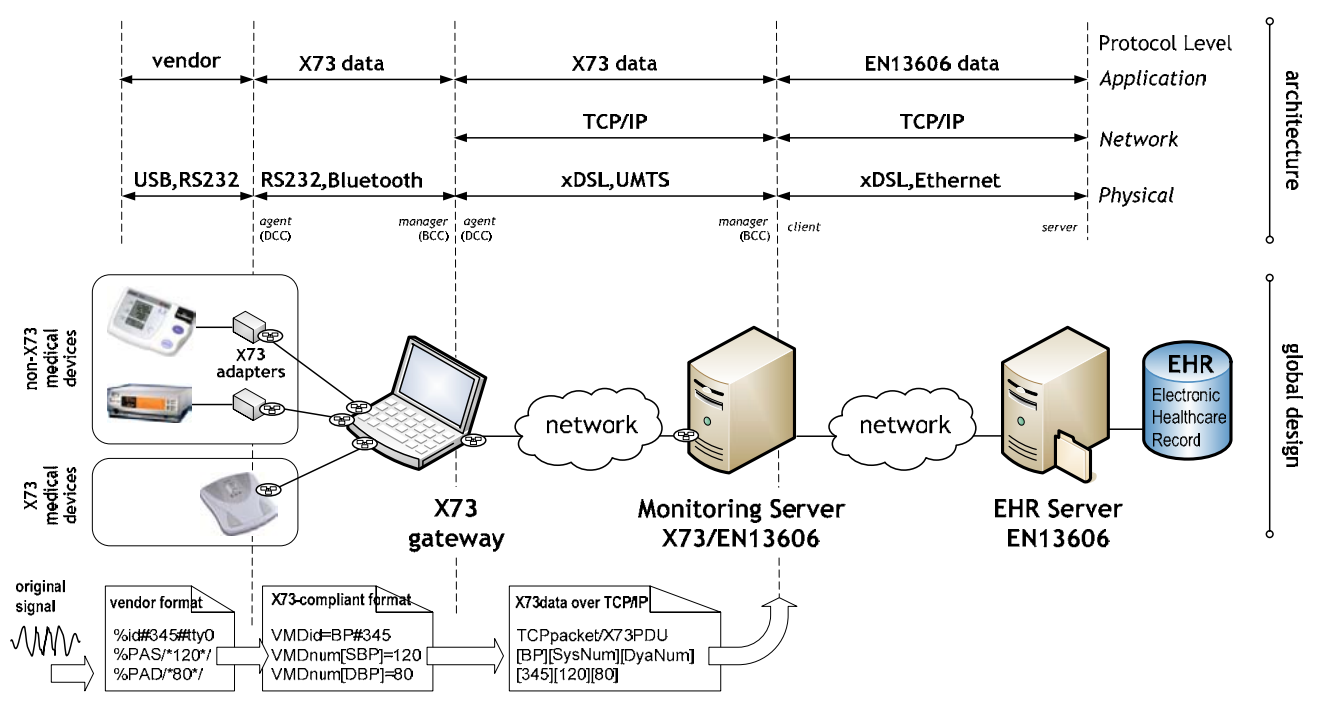

Fig. 1. Proposed end-to-end X73/EN13606 design scheme

\section{A. X73 MDs and adapters}

Currently there are no $100 \%$ compliant X73 MDs. In addition to this, it is difficult to find MDs with an X73compatible physical output noticing that only RS-232 and IrDA are included in the X73 standards at the moment. Thus, in this development, proprietary MDs have been used together with an X73-adapter which, on one hand implements the standard X73 and on the other hand, proprietary interfaces. These MDs are: OMRON 705IT (blood-pressure and pulse measurements with a USB output) and DATEX-Ohmeda 3900 (pulse oximeter with a serial-port output). This adapter includes the main X73 modules: Medical Device Data Language (MDDL), Domain Information Model (DIM), and Dynamic Model (that provides the communication model (agent-manager) from 'Device Communication Controller' (DCC) to 'Bedside Communication Controller' (BCC) for interconnection between X73 adapter and X73 gateway).

\section{B. X73 gateway}

The designed gateway is an X73 access point. It supports a network of X73-compatible MDs measuring vital signs from different patients in different locations. The data acquired from the different sensors is transmitted to the X73 gateway which provides a logic module for the Medical Data Information Base (MDIB). This module allows monitoring and alert and measurement acquisition. Thus, following the X73 nomenclature, this gateway is represented as a Medical Device System (MDS): when each MDS is either connected or disconnected (plug-and-play) the MDIB is automatically updated and a MDS is created in the object hierarchy.

\section{X73/EN13606 monitoring server}

The monitoring provides a double role: manager for the communications with the X73 gateways, and client for the connections to the EHR server. Thus, it may belong to the ICU, the reference hospital, or can even be used as an external management server.

As a manager for the X73 gateways, the monitoring server manages the decision making in an intelligent way through several functions: automation of the data acquisition process, technological transparency (independent of the transmission modes of the X73 gateways), definition of patterns to identify, detection and management of anomalies (alarms, failures), patient specifications (decision thresholds, operation limits), updates and evolutions, etc.

As a client for the EHR server, the monitoring server creates an EN13606 extract (from X73 data of the MDs) that is transmitted to the server in an intermediate archetype file.

\section{EN13606 EHR server}

The EHR server is a clinical information container that stores the databases that are associated with the EHR of each patient and updates them by incorporating the incoming information from the MDs in an EN13606 compliant format. Thus, it receives the archetype files with the EN13606 extract, validates this extract according to EN13606 standard, stores it in the appropriate EHR database, and sends back an acknowledgement to the client. This process traditionally has been studied in an isolated way as an EHR communications interoperability problem, but in this implementation experience it is integrated with the monitoring process to propose a entire end-to-end solution. 


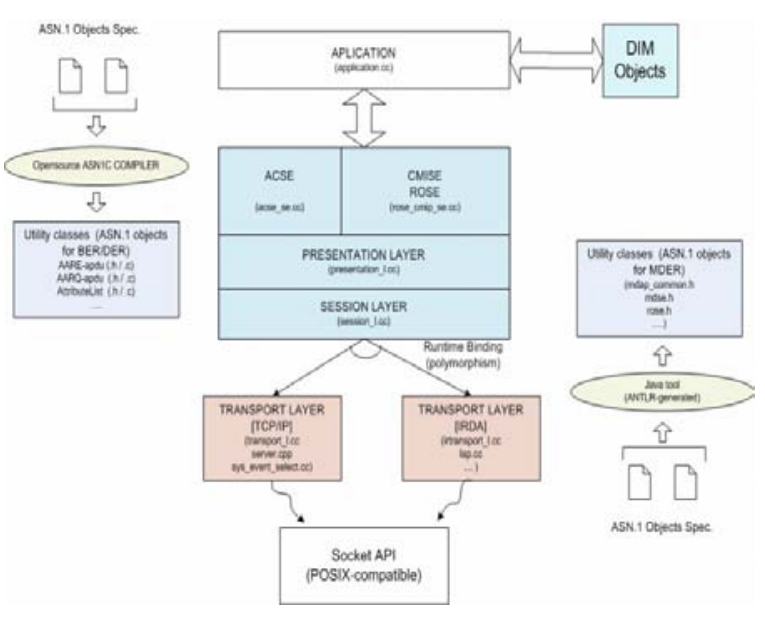

(a) Protocol stack scheme

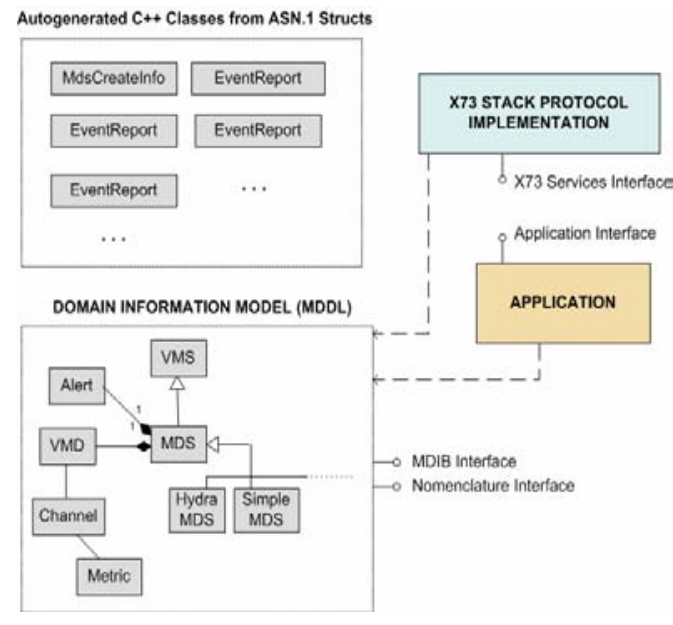

(b) Block diagram

Fig. 2. X73 gateway and monitoring server implementation scheme

\section{X73/EN13606 IMPLEMENTATION.}

\section{A. X73 implementation experience.}

The complete implementation experience scheme is shown in Fig. 2. The programming framework basically consists of Java, C/C++ and Abstract Syntax Notation One (ASN.1) as a language for the exchange of messages coded with Medical Device Encoding Rules (MDER, proposed codification rules for X73).

Following a downwards process through the protocol stack, firstly, the application layer is defined by several protocols: ACSE (for association control), CMISE (for the basic services defined in VITAL) and ROSE (for the link between call requests and responses). Both ROSE and CMISE are merged into CMDISE. Secondly, the presentation layer is mainly a negotiation mechanism for the syntaxes used by higher layers: the abstract syntax to use (which set of messages are to be exchanged) is specified by MDDL, and the transfer syntax (how the messages are encoded) is described by the MDER. Thirdly, the session layer provides support to the ACSE (a simplified version). Finally, the implementation of the transport layer varies regarding TCP/IP or IrDA-cable protocol being in our case TCP/IP over RS-232 transmission. The implementation details are described as follows, step-by-step according to the message exchange among the different layers and using implementation scheme:

1) The DCC controller (agent) initiates a connection to the BCC controller (manager): stack->transport->t_con_req(conn);

2) The BCC receives a connection request. It begins accepting incoming events and passes the request up to the application layer. The BCC starts an association request to the DCC.

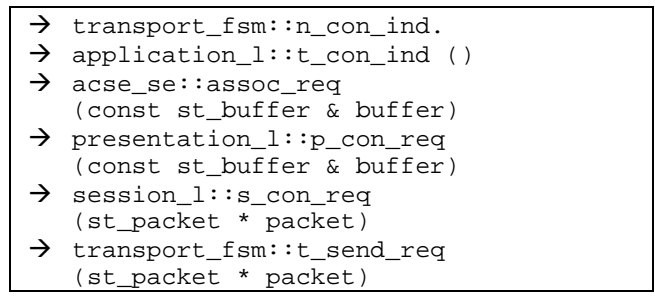

3) The DCC receives an association request and sends a confirmative answer:

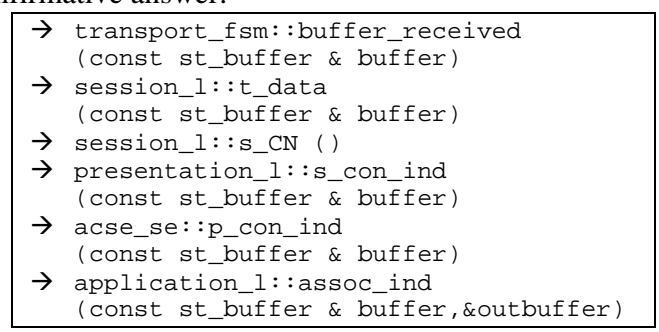

4) The BCC receives the association confirmation:

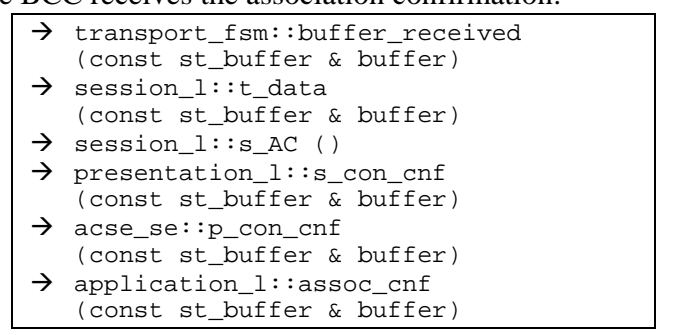

5) The connection procedure finishes. Now the entities exchange messages in conformance with the ROSE/CMIP protocol and ruled by the selected application profile (baseline or polling mode). 


\section{B. EN13606 EHR server implementation.}

The proposed implementation experience is completed with the process of storing medical device information in the EHR server, fulfilling EN13606 standard. The client/server architecture has been implemented with middleware technologies, based on Web Services (WS). The employed tools are Apache Axis (open source), the Servlets/JSPs container Apache Tomcat, eXtensible Mark-up Language (XML, WS-based framework based on an implementation of the SOAP server), and various utilities and APIs for generating and deploying Web service applications. Data are transmitted using HTTPS protocol and, in order to gain security, the Apache WSS4J framework is used.

On the client side, a Java application has been developed. Its function is to read the MD data which is stored in an intermediate archetype file (XML document), by giving the EN13606 standard format using eXtensible Style sheet Language Transformations (XSLT) and making the call to the WS to store this information in the EHR. On the server side, a WS has been implemented. Its functionality is to receive the XML extract, in EN13606 format, sent by the client and to validate it, using XML Schemes. Once the $\mathrm{XML}$ extract is validated, an acknowledgement will be sent to the client. If it was successful, the extract will be stored in the EHR Server database.

\section{DISCUSSION AND CONCLUSIONS}

The adoption of a complete standard end-to-end solution for patient monitoring inside hospitals can be extremely useful for the integration and interoperability of the huge amounts of vital data collected every day, making a more efficient use of such information, allowing it to be shared among professionals, giving more facilities for the mobility of patients within the hospitals, reducing costs and increasing usability for patients, health care professionals and manufacturers.

From this implementation experience it was seen that X73 implementation is feasible and the standard documents are reasonably understandable, however, some guidelines could be of great help for developers that want to apply standards to a specific system. In this case, and without previous experience, the implementation of the two standards in the whole system took no more than 3 months and it was performed by a group of 5 soft/hardware developers following the advice of the rest of the research group.

Some parts of the X73 standard are still subject to changes, and some layers are being adapted to improve its performance with different types of transmission technologies. The EN13606 standard is also evolving and changes in the domain information model are expected.

Home telemonitoring has experienced an important growth in the past years, and nowadays it has proved its efficiency as a follow-up option in scenarios such as chronic disease management, home hospitalization, follow-up after ambulatory surgery, and elderly patients' care. Mobile telehealth solutions are expanding the limits of telemonitoring, allowing patient follow up while they carry out the activities of their daily living. The work presented in this paper can be adapted for the implementation and design of a standard end-to-end solution for medical device communications in home and ambulatory environments, having an important impact in this promising market. Standards-based telemonitoring devices are critical for the eHealth sector, as they can foster competitiveness between manufacturers and help service providers in a definitive adoption of telemedicine.

\section{ACKNOWLEDGMENT}

The authors wish to thank Mr. Melvin Reynolds, convenor of the CEN TC251 WGIV, for his key suggestions to this research, Adolfo Muñoz for his technical support in the EHR server implementation, David Tejada and Rosario Achig for their help on software/hardware system development. We also appreciate the contribution of the Research Groups of the Spanish Telemedicine Research Network to the excellent results of the entire project carried out during the last four years.

\section{REFERENCES}

[1] C. Meyer, "Visions of tomorrow's ICU," American Journal of Nursing, vol. 93, pp. 26-31, 1993.

[2] T. D. East, "Computers in the ICU: Panacea or plague?," Respiratory Care, vol. 37, pp. 170-180,1992.

[3] P. S. Barie, "Advances in critical care monitoring," Archives of Surgery, vol. 132, pp. 734-739, 1997.

[4] F. Uckert, M. Ataian, M. Görz and H. U. Prokosch, "Functions of an electronic health record," International Journal of Computerized Dentistry, vol. 5, pp. 125-132, 2002.

[5] M. F. O'Toole, K. S. Kmetik, H. Bossley, J. M. Cahill, T. P. Kotsos, P. A. Schwamberger and V. J. Bufalino, "Electronic health record systems: the vehicle for implementing performance measures." The American Heart Hospital Journal, vol. 3, pp. 88-93, 2005.

[6] F. Chiarugi, D. Trypakis, M. Spanakis, "Problems and Solutions for Storing and Sharing Data from Medical Devices in e-Health Applications”, 2nd OpenECG Workshop, pp. 65-69, 2004.

[7] W. W. Stead et al., "Integration and beyond: Linking information from disparate sources and into workflow," Journal of the American Medical Informatics Association, vol. 7, pp. 135-145+146, 2000.

[8] S. Pedersen et al., "Interoperability for information systems among the health service providers based on medical standards," Informatik Forschung Und Entwicklung, vol. 18, pp. 174-188, 2004.

[9] R. J. Kennelly, "Improving acute care through use of medical device data," Int J Med Inform, vol. 48, pp. 145-149, 1998.

[10] P. de Toledo, M. Galarraga, I. Martinez, L. Serrano, J. Fernández, F. del Pozo, "Towards e-Health Device Interoperability: The Spanish Experience in the Telemedicine Research Network", Int Conf IEEE Eng in Medicine and Biology Society, EMBS, pp. 3258-59, 2006.

[11] IEEE1073. Health informatics. Point-of-care medical device communication. Standard for Medical Device Communications Overview and Framework. http://www.ieee1073.org.

[12] ENV13606-CEN/TC251. Electronic Healthcare Record Comm. Parts 1, 2, 3 and 4, Pre-standard, 2000," http://www.medicaltech.org.

[13] M. Galarraga, L. Serrano, I. Martinez and P. de Toledo, "Standards for Medical Device Communication: X73 PoC-MDC", Medical and Care Compunetics 3. IOS Press - "Studies in Health Technology and Informatics" vol. 121, pp. 242-256, 2006. 